Revista Mexicana de Economía y Finanzas Nueva Época Volumen 14 Número 3, Julio-Septiembre 2019, pp. 447-457

DOI: https://doi.org/10.21919/remef.v14i3.409

\title{
Estimación de la distribución multivariada de los rendimientos de los tipos de cambio contra el dólar de las criptomonedas Bitcoin, Ripple y Ether
}

\author{
Beatriz Mota Aragón ${ }^{1}$ \\ Universidad Autónoma Metropolitana, México \\ José Antonio Núñez \\ Tecnológico de Monterrey, México
}

(Recepción: 28/noviembre/2018, aceptado: 1/marzo/2019)

\section{Resumen}

En este artículo se estima la distribución multivariada para analizar la dependencia del Bitcoin (BTC), Ripple (XRP) y Ether (ETH). Se utiliza la familia Hiperbólica Generalizada de distribuciones $(\mathrm{GH})$ y en particular la distribución Varianza Gamma. El procedimiento para la estimación de los parámetros de la GH es a través del algoritmo EM (Expectation-Maximization). Los resultados muestran que existe una dependencia positiva entre los tres tipos de cambio respecto del dólar americano y se estima una distribución Varianza-Gamma de dimensión tres. Esta distribución es muy flexible para el ajuste de series de los rendimientos con leptocurtosis y sesgo. Esta información se considera importante para los inversionistas que conforman sus portafolios de una manera eficiente.

Clasificación JEL: C11, C46

Palabras clave: leptokurtosis, asimetria, varianza gamma, multivariante

\section{Estimation of the multivariate distribution of exchange rate yields against the dollar of the Cryptocurrencies Bitcoin, Ripple and Ether}

\section{Abstract}

In this paper we estimated the multivariate distribution among Bitcoin (BTC), Ripple (XRP) and Ether (ETH) to analyze the dependence. We used the Hyperbolic Generalized (GH) family of distributions and in particular the Variance-Gamma distribution. The procedure for the estimation of the parameters of the $\mathrm{GH}$ distribution is through the EM (Expectation-Maximization) algorithm. The results show that there exists a positive dependence among the three exchange rates with respect to the American dollar and a Variance-Gamma distribution of dimension three is estimated. This distribution is very flexible for the adjustment of returns with leptokurtosis and skewness. This distribution is very flexible for the adjustment of the returns with leptokurtosis and skewness. The information is important for the investors who construct their portfolios in an efficient way.

JEL Classification: C11, C46

Keywords: leptokurtosis, skewness, Variance-Gamma, Multivariate

\footnotetext{
${ }^{1}$ beatrizmota4@gmail.com

*Sin fuente de financiamiento declarada para el desarrollo de la investigación
} 
REMEF (The Mexican Journal of Economics and Finance)

Estimación de la distribución multivariada de los rendimientos de los tipos de cambio contra el dólar de las criptomonedas Bitcoin, Ripple y Ether

\section{Introducción}

La tecnología blockchain y las criptomonedas han llamado la atención de una manera importante en la última década. De entre las más sobresalientes están el Bitcoin, que en la literatura es común encontrarla como la primera criptomoneda digital descentralizada, la cual desde su creación utiliza un conjunto de funciones criptográficas; sin embargo, en orden cronológico fue el eCash, el primer sistema criptográfico monetario electrónico (Chaum, 1983) y más tarde, el e-Gold el cual permitió la posibilidad de hacer transferencias instantáneas de valor (GSR, 1998), Henriques, et al. (2018). El Bitcoin creado por Satoshi Nakamoto en 2009, alcanzó la capitalización de mercado en abril del 2018 arriba de los 116 billones de dólares (coinmarketcap.com), y en general la capitalización del mercado de criptomonedas asciende los 295 billones de dólares con más de 1600 (Shuyue et. al. 2018) de ellas que han surgido con diferentes propiedades. Las características distintivas del Bitcoin son que ninguna autoridad central la respalde o controle y que su oferta está limitada por el diseño del protocolo, Bouri et al. (2017) y Dwyer (2015). Por su parte el Ether, la primera moneda basada en tecnología de una cadena de bloques o blockchain; por último, el Ripple distinta del bitcoin debido a que no utiliza el proceso de minería, sino que las transacciones se verifican entre los miembros de la red, son también criptodivisas destacables. Estos novedosos activos tienen nuevas características que los hacen únicos; tienen un control descentralizado en su operación y en su transacción. El uso del cryptocash es distinto al tradicional uso de efectivo, lo cual indica que las transacciones pueden ser realizadas directamente entre agentes económicos sin necesidad de intermediación financiera y control de cambios, lo cual reduce los costos de transacción y los tiempos. En Sahoo (2017), se discute el futuro del Bitcoin como posible medio de cambio, y se concluye que esta sobrevivencia está sujeta principalmente a que tenga una volatilidad más moderada en el futuro, ya que esto originaría una mayor confianza en los usuarios de dicha criptomoneda. Aunque con datos diarios la volatilidad de los rendimientos del Bitcoin es mayor que los del oro y otras monedas en dólares americanos, es interesante el hallazgo en Dwyer (2015), encuentra que en ciertos periodos de tiempo, las volatilidades mensuales del Bitcoin son menores. De esta manera, no queda claro que de manera uniforme los rendimientos del Bitcoin sean mayores siempre en cualquier circunstancia.

Nuestro trabajo se interesa especialmente en estudiar la dependencia multivariada de los rendimientos de los tipos de cambio de las criptomonedas Bitcoin, Ripple y Ether contra el dólar, y contribuye al estudio y desarrollo de una explicación teórica de la mencionada dependencia.

La investigación se realiza a través de la familia hiperbólica generalizada (GHD) multivariada. En particular, se encuentra que con una distribución Varianza-Gamma de orden tres, es posible describir una estructura de dependencia positiva. El enfoque aplicado corresponde al de distribuciones paramétricas con una relación entre las características de los datos (hechos estilizados) y los momentos de la distribución, que están en función de los parámetros calculados.

Los resultados empíricos proveen la utilidad del trabajo. Por otro lado, planteamos como Corbet et al. (2018b), Glaser et al. (2014), Demir et al. (2018) la naturaleza de las criptomonedas como un medio de cambio e instrumento de inversión especulativo, en este sentido tiene implicaciones en la teoría de portafolios y la teoría de administración de riesgos y reconocemos la naturaleza dual de las criptomonedas, y la determinación de la tecnología y de los factores económicos como una línea de investigación futura, Gandal Halabrurda (2016), Xin, et al. (2016). De manera interesante existen investigaciones que no encuentran que la volatilidad del Bitcoin sea una consecuencia de la especulación, como puede verse en Blau (2017). Los resultados de esta investigación contribuyen a la comprensión del mercado altamente dinámico de las criptomonedas. El trabajo se divide 
en cinco secciones: En la sección dos se hace la revisión de literatura, en la tres se explica la metodología y los datos. En la sección cuatro exponemos los resultados del estudio, y la sección cinco se refiere a las conclusiones.

\section{Revisión de literatura}

Los factores que intervienen en la formación de precios del Bitcoin son radicalmente diferentes a los de activos convencionales. Estos factores incluyen noticias en redes, búsquedas en internet, Kristoufek (2013), tendencias en google (García et al., 2014), entre otras. La naturaleza del Bitcoin de acuerdo a Yemack (2013) es especulativa, y aunque en el mercado las principales criptomonedas son el Bitcoin y el Ripple, se sabe que la capitalización y la proporción del mercado de las criptomonedas ha variado fuertemente junto con su volatilidad (White, 2014). También Glaser et al. (2014) encuentra que la mayoría de los inversionistas en Bitcoin lo tratan con un fin especulativo, de lo cual deduce que es más un activo que una moneda. En Baur et al. $(2017,2018)$ consideran que el Bitcoin es un intermedio entre un metal precioso y una moneda fiduciaria. De manera interesante se establece que tiene una correlación débil o nula con activos tradicionales (acciones, bonos, oro etc.), y por tanto puede utilizarse como opción de diversificación en un portafolio de inversión.

En el sentido anterior, Dyhrberg $(2015,2016)$ estudia la capacidad de cobertura del Bitcoin contra los tipos de cambio del dólar americano con el Euro y la libra esterlina y concluye que es un valioso activo para períodos de crisis para un perfil de inversionista adverso al riesgo, teniendo estas características intermedias entre el oro y el dólar americano; es decir, entre la característica de una moneda fiduciaria como medio de cambio y la capacidad de guardar valor del oro. Es de notarse la falta de liquidez del Bitcoin con respecto a otros activos, y la presencia de correlación serial en la serie de sus rendimientos. El análisis econométrico se llevó a cabo con un GARCH asimétrico, y el caso del Bitcoin se halló que genera mayor volatilidad cuando hay períodos de crisis y disminuye cuando hay noticias buenas, lo cual es lo contrario de lo que sucede con acciones. Sin embargo, Baur et al. (2017) replica el estudio de Dyhrberg (2016) y encuentra que los rendimientos del Bitcoin siguen un proceso de volatilidad diferente a otros activos como el oro, y demuestra que se encuentra no correlacionado con otros activos como el dólar americano y el mismo oro. Respecto al estudio de las correlaciones, en Bouri et al. (2017) se analizan las propiedades de cobertura y de refugio (como el oro) del Bitcoin, usando un modelo de correlaciones condicionales dinámicas (DCC). Los autores concluyen que el Bitcoin es un activo útil para la diversificación, aunque esta propiedad varía con el tiempo, así como también las propiedades de ser un activo para cobertura y refugio seguro varían dependiendo del periodo de tiempo estudiado.

Como es conocido, la modelación de la volatilidad en finanzas es uno de los aspectos más importantes pues interviene en la administración del riesgo, en la formación de portafolios, en la estructura de formación de burbujas especulativas entre otros aspectos. La modelación de la volatilidad es uno de los aspectos más discutidos recientemente en la literatura. Katsiampa (2017) usó el modelo AR-CGARCH para estimar la volatilidad del Bitcoin y Urquhart (2017) concluyó que los modelos HAR son mejores en estimar la volatilidad del Bitcoin que los modelos GARCH usuales.

Complementando lo anterior también se ha trabajado en relacionar variables macroeconómicas con la volatilidad del Bitcoin. Cemak (2017) usó un GARCH $(1,1)$ para países con alto volumen de trading. Concluye que el Bitcoin se porta de manera similar a las monedas fiduciarias en China, Estados Unidos y Europa, pero no en Japón. En Chu et al. (2017) modelan la volatilidad de siete criptomonedas con doce modelos tipo GARCH, a decir, SGARCH, EGARCH, GJRGARCH, APARCH, IGARCH, CSGARCH, GARCH, TGARCH, AVGARCH, NGARCH, NAGARCH y ALLGARCH para el Bitcoin, Dash, Dogecoin, Litecoin, Maidsafecoin, Monero y Ripple. Los mejores modelos de acuerdo con 
estos autores fueron el IGARCH y el GJRGARCH, en donde es de destacar que el crecimiento del Bitcoin, Ether, Litecoin y Riple ha sido mayor que el resto de las criptomonedas. Los autores plantean en sus conclusiones que es necesario investigar y desarrollar el comportamiento multivariado de los tipos de cambio del Bitcoin, Dogecoin, Maidsafecoin, Monero y Ripple. En contraste, en Katsiampa (2017) se explica que la inclusión de las componentes de corto y largo plazo en la modelación de la volatilidad condicional, se encuentra que el modelo AR-CGARCH es el que mejor ajusta los datos del rendimiento del Bitcoin.

Además de las características antes mencionadas del Bitcoin y de las criptomonedas, los enfoques de econofísica han contribuido para ir más a fondo en el estudio de los orígenes de las burbujas y caídas desde la perspectiva monetarista, ver Corsi and Sornette (2014). Las burbujas y caídas para el caso de las criptomonedas tienen un origen diferente y representa un reto importante para la economía y los mercados financieros el descubrir su naturaleza. Las burbujas especulativas son estudiadas en Fry y Chea (2016) desde el enfoque de la econofísica, demostrando primeramente la utilidad de estas herramientas en el estudio del mercado de las criptomonedas y también sugieren su potencial uso en la cobertura y trading en el mercado financiero. Katsiampa (2017) menciona igualmente que el uso del Bitcoin como activo más que como moneda, lo hace especulativo y volátil, así como más susceptible a la generación de burbujas que las monedas. Sin embargo, en Blau (2017) se encuentra que la volatilidad de los rendimientos del Bitcoin no es consecuencia de la especulación con dicha criptomoneda, en el periodo de 2010 a 2013. El 2013 es un punto de referencia importante pues el valor del Bitcoin comenzó con un valor de 13hastallegaramsde1000. El anterior estudio está basado en una medida de trading especulativo propuesta por Llorente et al. (2002).

Otra herramienta que es fundamental en diversas áreas de finanzas como la administración de riesgos financieros, la formación de portafolios de inversión, la simulación Monte Carlo, entre otras, es la estimación de la distribución de probabilidad. En Chu et al. (2015) se encuentra que la distribución que mejor se ajusta a los datos del rendimiento del Bitcoin vs. Dólar americano, es la hiperbólica generalizada, la cual tiene un excelente grado de flexibilidad debido a que usan cinco parámetros.

Adicional a lo anterior, Chan et al. (2017) estudian los rendimientos de tipo de cambio contra el dólar americano de las seis principales criptomonedas (de acuerdo a la capitalización del mercado son el Bitcoin, Dash, Dogecoin, Litecoin, MaidSafeCoin y Ripple), y se estiman las distribuciones paramétricas.

Los rendimientos de las criptomonedas exhiben muy alto valor de la curtosis y sesgo negativo a excepción del Bitcoin y el Litecoin. Las distribuciones encontradas son la hiperbólica generalizada, la normal inversa Gaussiana, t Generalizada y Laplace, todas ellas univariadas. Es decir, para este tipo de rendimientos existen colas pesadas que determinan de manera importante valores como la Valor en Riesgo y el VaR condicionado, así como la matriz de varianzas y covarianzas. En otro estudio, Zhang et al. (2018) revisa los hechos estilizados de los rendimientos del Bitcoin, Ether, Ripple, Litecoin, Stellar, Dash, Monero y NEM, donde se destaca el hecho de que todas tienen una curtosis mayor a 3 y la distribución no es normal al 1\% de nivel de significancia. Las autocorrelaciones para los rendimientos absolutos decaen lentamente, y se prueban los fenómenos de apalancamiento con el modelo GJR(1,1) y cúmulos de volatilidad con un modelo $\operatorname{GARCH}(1,1)$. Es importante estudiar las distribuciones multivariadas de los rendimientos de diferentes criptomonedas, ya que se puede encontrar que existe una dinámica entre ellas, que es significativa, y que por tanto tiene un impacto importante en el diseño de portafolios y medición del riesgo entre otros aspectos. 


\section{Metodología y Datos}

\section{Datos}

Los datos seleccionados son los rendimientos diarios de las criptomonedas Bitcoin, Ripple y Ether del 02 de febrero del 2016 al 15 de octubre de 2018, de investing: https://mx.investing.com/. Se calculará la distribución multivariada de dimensión tres.

\section{Distribución de probabilidad y prueba de hipótesis}

La distribución hiperbólica generalizada (GHD) tiene parámetros que describen los hechos estilizados de los rendimientos, en este caso lo aplicamos a los rendimientos de los tipos de cambio de criptomonedas contra el dólar (Protassov 2004 y Paolella 2007). La distribución se caracteriza por cinco parámetros en la función de densidad:

$$
f(x ; \lambda, \alpha, \beta, \delta, \mu)=\frac{k_{\lambda-12}\left((x-\mu)^{2}+\delta^{2}, \alpha^{2}\right)}{\sqrt{2 \pi} k_{\lambda}\left(\delta^{2}, \alpha^{2}-\beta^{2}\right)} \exp [\beta(x-\mu)],
$$

con las restricciones $\alpha, \delta \geq 0, \lambda, \mu, \beta \in \mathbb{R}$ y $\beta \in[-\alpha, \alpha]$ y la función de Bessel de tercer orden,

$$
K_{\lambda}(x)=\frac{1}{2} \int_{0}^{\infty} t^{\lambda-1} e^{-\frac{1}{2} x\left(t+t^{-1}\right)} d t .
$$

donde $\lambda \in \mathbb{R}$ y $x>0$. La función de densidad de una variable aleatoria Gaussiana Inversa Generalizada (GIG), ver Barndorff-Nielsen (1977) es:

$$
\begin{aligned}
f(x ; \lambda, \chi, \psi)=\frac{1}{k_{\lambda}(\chi, \psi)} & x^{\lambda-1} \exp \left[-\frac{1}{2}\left(\chi x^{-1}+\psi x\right)\right], \\
k_{\lambda}(\chi, \psi) & =2 \eta^{\frac{\lambda}{2}} K_{\lambda}(\omega) \\
\eta & =\chi / \psi \\
\omega & =\sqrt{\chi \psi}
\end{aligned}
$$

Los parámetros $\lambda \in \mathbb{R}, \chi \geq 0$ y $\psi \geq 0$ determinan la localización y la dispersión de $x \in \mathbb{R}_{+}$,

También con los siguientes cambios,

$$
\begin{gathered}
\chi:=\delta^{2} \\
\psi:=\alpha^{2}-\beta^{2}
\end{gathered}
$$

Que el valor esperado

$$
E[X]=\mu+\beta \frac{k_{\lambda+1}(\chi, \psi)}{k_{\lambda}(\chi, \psi)}
$$

Y la varianza

$$
V[X]=\frac{k_{\lambda+1}(\chi, \psi)}{k_{\lambda}(\chi, \psi)}+\beta^{2} \frac{k_{\lambda}(\chi, \psi) k_{\lambda+2}(\chi, \psi)-\left[k_{\lambda+1}(\chi, \psi)\right]^{2}}{\left[k_{\lambda}(\chi, \psi)\right]^{2}}
$$

Para el caso de una distribución hiperbólica generalizada multivariada, tomemos un vector aleatorio $\mathrm{X}$ de tamaño $n \times 1$ y diremos que sigue una función de densidad hiperbólica generalizada multivariada $(\mathrm{GH})$, si esta función se expresa como

$$
f(x ; \lambda, \gamma, \beta, \mu, \delta, \Delta)=\frac{\left(\sqrt{\frac{\delta^{2}+(x-\mu) \Delta^{-1}(x-\mu) \prime}{\gamma^{2}+\beta^{\prime} \Delta \beta}}\right)^{\lambda-\frac{n}{2}}}{(2 \pi)^{\frac{n}{2}}\left(\frac{\gamma}{\delta}\right)^{-\lambda}} \frac{K_{\lambda-\frac{n}{2}} \sqrt{\delta^{2}+(x-\mu) \Delta^{-1}(x-\mu) \prime\left(\gamma^{2}+\beta \prime \Delta \beta\right)}}{K_{\lambda}(\delta \gamma) \exp (-(x-\mu) \beta \prime)}
$$

con $\chi=\delta^{2}$ y $\psi=\gamma^{2}-\beta \prime \Delta \beta$, los parámetros de una variable GIG. También $X \mid W \sim N(\mu+w \beta \Delta, w \Delta)$

$W \sim G I G(\lambda, \chi, \psi)$ 
La matriz de covarianzas para el vector aleatorio X es $V[X]=E[W] \Delta+V[W] \beta^{\prime} \beta$ y el valor esperado es $E[X]=\mu+E[W] \beta(\mathrm{Hu}, 2005)$.

Respecto a la interpretación de los parámetros tenemos que $\lambda$ está relacionado con qué tan planas son las colas de la distribución, $\chi$ y $\psi$ de manera conjunta determinan la forma de la distribución (por ejemplo cuando estos parámetros toman valores grandes en su dominio, la distribución se aproxima a la normal), $\mu$ nos dice dónde está centrada la distribución, y finalmente $\gamma$ es el parámetro que indica el sesgo, Konlack y Wilcox (2014)

\section{Estimación}

El procedimiento para la estimación de los parámetros de la GH es a través del algoritmo EM (Expectation-Maximization), ver Protassov (2004) y Breymann (2013). En particular, la distribución Varianza-Gamma también puede verse de la siguiente manera:

$$
f(x)=c(c, \sigma, \theta, \vartheta)|x-c|^{1 / \vartheta-1 / 2} e^{\left\lceil\theta(x-c) / \sigma^{2}\right\rceil} K_{1 / \vartheta-1 / 2}\left(\frac{|x-c| \sqrt{\frac{2 \sigma^{2}}{\vartheta}+\theta^{2}}}{\sigma^{2}}\right)
$$

donde $K_{\vartheta}$ es la función de Bessel de tercer orden,

$$
c(c, \sigma, \theta, \vartheta)=\frac{2}{\sigma \sqrt{2 \pi} \vartheta^{1 / \vartheta} \Gamma(1 / \vartheta)}\left(\frac{1}{\sqrt{\frac{2 \sigma^{2}}{\vartheta}+\theta^{2}}}\right)^{\frac{1}{\vartheta}-1 / 2}
$$

\section{Estimación de la función de densidad}

Si el vector de parámetros está definido como $\Theta=(\lambda, \gamma, \delta, \beta, \mu, \Delta)$, el logaritmo de la función de verosimilitud es

$$
\ln \left(L\left(\Theta ; x_{1}, \ldots, x_{m}\right)=\sum_{i=1}^{m} \ln \left(f\left(x_{i} ; \Theta\right)\right)\right.
$$

donde $x_{1}, \ldots, x_{m}$ es una muestra de vectores de números aleatorios. El algoritmo EM considera variables latentes $w_{1}, w_{2}, \ldots, w_{m}$ con una distribución GIG. La función de verosimilitud expandida es

$$
\begin{gathered}
\ln \left(L^{\prime}\left(\Theta ; x_{1}, \ldots, x_{m}, w_{1}, w_{2}, \ldots, w_{m}\right)\right)=\sum_{i=1}^{m} \ln \left(f_{X \mid W}\left(x_{i} \mid w_{i} ; \mu, \Delta, \beta\right)\right)+ \\
\sum_{i=1}^{m} \ln \left(f_{W}\left(w_{i} ; \lambda, \gamma, \delta\right)\right.
\end{gathered}
$$

Después de la selección de un punto inicial $\Theta_{0}$, se desarrollan los siguientes pasos:

a) Estimación del valor esperado de la función del logaritmo de la función de máxima verosimilitud aumentada,

$h\left(\Theta ; \Theta_{k}\right)=E\left[\ln \left(L^{\prime}\left(\Theta ; x_{1}, \ldots, x_{m}, w_{1}, w_{2}, \ldots, w_{m}\right)\right) \mid x_{1}, \ldots, x_{m} ; \Theta_{k}\right]$

b) Maximizar la función objetivo $h\left(\Theta ; \Theta_{k}\right)$ para obtener la aproximación

$\Theta_{k+1}$ hasta que $\left|\Theta_{k+1}-\Theta_{k}\right|<\varepsilon$.

$\mathrm{El}$ anterior algoritmo se programó en el paquete R. Se probaron diversos puntos iniciales del método numérico, entre ellos la media y mediana de cada serie de datos. El mismo programa ghyp en $\mathrm{R}$ realiza diversas pruebas con valores iniciales, Breymann Lüthi (2013).

Se puede demostrar que el estimador $\hat{\Theta}$ tiene distribución normal multivariada asintótica, es decir,

$$
\sqrt{m}\left(\hat{\Theta}_{i}-\Theta_{i}\right) \rightarrow N\left(0, I^{-1}\right)
$$

donde

$$
\begin{gathered}
I=\operatorname{Cov}\left(S_{i}\right) \\
S_{i}=\frac{\partial \ln \left[f\left(x_{i} ; \hat{\Theta}_{i}\right)\right]}{\partial \hat{\Theta}_{i}}
\end{gathered}
$$


El intervalo para el estimador (Barndorff-Nielsen, 2012), $\hat{\Theta}_{i}$ es

$$
\begin{gathered}
\hat{\Theta}_{i} \pm t_{\alpha / 2} \sqrt{\frac{1}{m}\left(I^{-1}\right)_{\hat{\Theta}_{i} \hat{\Theta}_{i}}} \\
t=\frac{\hat{\Theta}_{i}-\Theta}{\sqrt{V\left[\hat{\Theta}_{i}\right]}}
\end{gathered}
$$

Para un nivel de significancia $\alpha$. La bondad de ajuste entre una muestra de datos y la GH multivariada se desarrolla mediante la prueba de McAssey (2013).

La prueba de McAssey consiste en dos pasos:

I) Simulación de una muestra aleatoria GH, $\left\{\hat{u}_{1}, \hat{u}_{2}, \ldots, \hat{u}_{T}\right\}$, con los parámetros estimados a partir de algoritmo EM (Ross, 2012).

II) Cálculo de la distancia de Mahalanobis entre la muestra de datos y a i) con la media muestral $\hat{\mu}$ y la matriz de covarianzas $\hat{\Sigma}$.

$$
\hat{d}_{i}=\sqrt{\left(\hat{u}_{i}-\hat{\mu}\right) / \hat{\Sigma}^{-1}\left(\hat{u}_{i}-\hat{\mu}\right)}
$$

Intuitivamente, la distancia de Mahalanobis coincide con la distancia Euclidiana si los vectores que estamos utilizando no están correlacionados. Por otro lado, si existe correlación entre los vectores, entonces se pondera por medio de la matriz de covarianzas $\hat{\Sigma}$, para obtener la distancia entre dichos vectores.

Por tanto, se obtiene una muestra aleatoria $\left\{A_{1}, A_{2}, \ldots, A_{p}\right\}$ con

$$
A_{i}=\sum_{j=1}^{T}\left|1-\frac{E_{j}}{O_{j}}\right|
$$

donde $E_{j}$ es la frecuencia esperada de las observaciones en un intervalo fijo, $O_{j}$ es la frecuencia observada en dicho intervalo y el valor p de la prueba es calculado.

\section{Resultados}

Para las series de tiempo de las criptomonedas Bitcoin, Ether y Ripple (rendimientos), se obtuvieron las estadísticas de la tabla 1. Es de notarse la alta curtosis de los rendimientos del Bitcoin y del Ripple. Existe un sesgo negativo en los tres casos.

Tabla 1. Estadística Descriptiva

\begin{tabular}{|lcccccc|}
\hline Variable & Promedio & $\begin{array}{c}\text { Desviación } \\
\text { Estándar }\end{array}$ & Sesgo & Curtosis & Mínimo & Máximo \\
\hline BITCOIN & 0.0029 & 0.0460 & -0.5880 & 10.2213 & -0.3716 & 0.3083 \\
ETHER & 0.0031 & 0.0631 & -0.0227 & 2.9306 & -0.3134 & 0.2586 \\
RIPPLE & 0.0026 & 0.1743 & -1.3621 & 85.3564 & -2.4079 & 2.3026 \\
\hline
\end{tabular}

Aplicando las pruebas de raíces unitarias obtenemos que ninguna de las series de rendimientos presenta raíces unitarias (Tabla 2). La gráfica de los rendimientos se muestra también (gráfica 1). Esta gráfica ciertamente confirma lo obtenido las pruebas de DickeyFuller, Phillips-Perron y KPSS del paquete EVIEWS. Por tanto, podemos continuar con el cálculo de los parámetros de la distribución de dimensión tres.

Tabla 2. Pruebas de Raíces Unitarias

\begin{tabular}{|lccc|}
\hline Variable & $\begin{array}{c}\text { Dickey Fuller } \\
\text { Estadístico t }\end{array}$ & $\begin{array}{c}\text { Phillips-Perron } \\
\text { Estadístico t }\end{array}$ & $\begin{array}{c}\text { KPSS } \\
\text { Estadístico t }\end{array}$ \\
\hline BITCOIN & $-49.33^{* * *}$ & $-49.34^{* * *}$ & 0.21 \\
ETHER & $-30.69^{* * *}$ & $-30.75^{* * *}$ & 0.35 \\
RIPPLE & $-17.30^{* * *}$ & $-17.65^{* * *}$ & 0.15 \\
\hline \multicolumn{4}{c}{$* * *$ Nivel de significancia de 1\%, } \\
& $* *$ : Nivel de significancia de $5 \%$, \\
& $*$ : Nivel de significancia de $10 \%$
\end{tabular}


REMEF (The Mexican Journal of Economics and Finance)

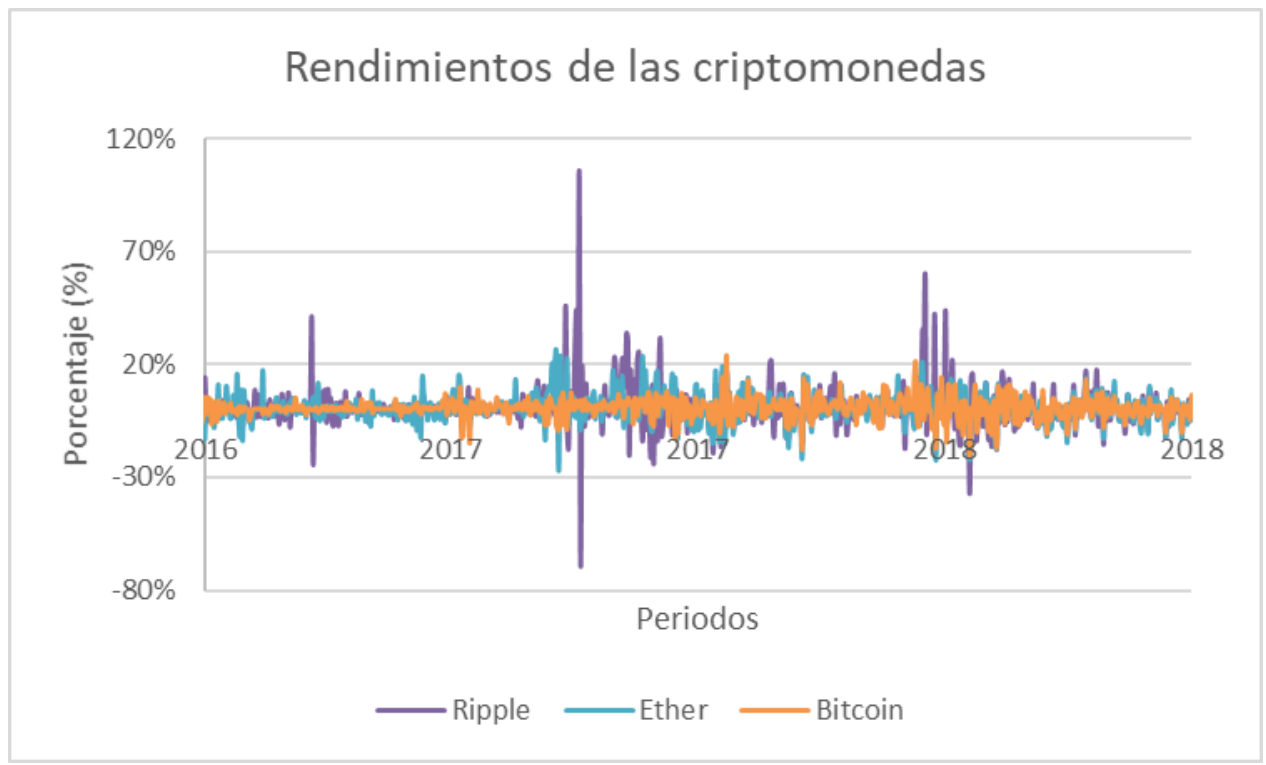

Figura 1.

Para la estimación de los parámetros se usó el algoritmo EM, de tal manera que una vez que se tienen los parámetros, probamos si la distribución es normal multivariada o Varianza-Gamma. De acuerdo con la prueba de McAssey (2013), se rechaza la hipótesis de normalidad de los datos y no se rechaza la hipótesis de una distribución VarianzaGamma, Tabla 3. Algunas de las ventajas que tiene la distribución Varianza-Gamma son que incluye colas más pesadas que las de la distribución normal y todos los momentos de la distribución son finitos entre otras propiedades, Madan y Seneta (1990). Las distribuciones varianza-gamma forman una subclase de la distribución hiperbólica propuesto por O.E. Barndorff-Nielsen (1977).

Podemos notar de la matriz de covarianzas de la distribución de orden 3, que existe una dependencia positiva lo cual nos proporciona información importante; por ejemplo, en la formación de portafolios, pues no podemos diversificar en gran medida con las tres criptomonedas, pues existe una relación en el mismo sentido. La diversificación de un posible portafolio se tiene que buscar a través de otros activos. También hay que decir que en este punto no podemos determinar que estas tres criptomonedas sean usadas para especulación solamente o al menos de manera importante.

Tabla 3. Estimación de los parámetros de la distribución de dimensión tres

\begin{tabular}{|cccc|}
\hline Log-verosimilitud & 4798.77 & $\lambda$ & $\begin{array}{c}0.7510 \\
(1.68)^{*}\end{array}$ \\
\hline$\mu$ & $\begin{array}{c}0.0026 \\
(-1.82)^{*}\end{array}$ & $\begin{array}{c}-0.0023 \\
(-1.73)^{*}\end{array}$ & $\begin{array}{c}-0.0026 \\
(1.93)^{*}\end{array}$ \\
\hline$\Delta$ & BITCOIN & ETHER & RIPPLE \\
\hline BITCOIN & 0.0019 & 0.0014 & 0.0012 \\
& $(1.96)^{* *}$ & $(1.83)^{*}$ & $(2.03)^{* *}$ \\
ETHER & 0.0014 & 0.0043 & 0.0020 \\
& $(1.83)^{* *}$ & $(2.05)^{*}$ & $(1.82)^{*}$ \\
RIPPLE & 0.0012 & 0.0020 & 0.0051 \\
& $(2.03)^{*}$ & $(1.82)^{*}$ & $(1.95)^{*}$ \\
\hline \multirow{2}{*}{$\gamma$} & 0.0004 & 0.0054 & 0.0069 \\
& $(1.96)^{* *}$ & $(1.88)^{*}$ & $(1.47)$ \\
\hline ***: Nivel de significancia de 1\%; ** : Nivel de significancia de 5\%; * : Nivel de significancia de 10\%.
\end{tabular}


Como se observa de la matriz de varianzas y covarianzas para la distribución de dimensión tres, resaltan las covarianzas positivas y pequeñas, lo cual tiene consecuencias importantes en términos de inversión. Por un lado, nos indica que habría limitaciones para invertir en las tres criptomonedas en un mismo portafolio, pues hay limitaciones para generar un portafolio diversificado. Por otro lado, las características de volatilidad de las tres criptomonedas puede deberse a una fuerte especulación en este mercado, aunque falta desarrollar estudios más profundos para investigar este último punto, ya que se ha visto para ciertos periodos de tiempo que la alta volatilidad característica no es necesariamente consecuencia de la especulación. La prueba de bondad de ajuste de MacAssey nos dice que los datos se ajustan a la distribución varianza gamma de una manera razonable. La normalidad de los datos es rechazada como era de esperarse para datos con colas pesadas, debido a la volatilidad existente.

\section{Conclusiones}

El surgimiento de las criptomonedas ha significado la aparición de lo que llamaríamos un activo y que tiene características de moneda. Sin embargo, no está clara su naturaleza económica y no se ha definido lo que es. A pesar de lo anterior, existe un mercado importante de las criptomonedas,- sobre todo el Bitcoin-, y es un gran reto analizar y modelar estadísticamente la dependencia de este con el Ripple y el Ether. Hemos encontrado una dependencia positiva y pequeña, a través de una distribución de colas pesadas. La matriz de correlaciones es un ingrediente determinante de la formación de portafolios a través de la diversificación. Las distribuciones Varianza-Gamma forman una subclase de la familia hiperbólica generalizada. En la literatura, puede ser que éstos sean utilizados con fines de diversificación, cobertura o como refugio seguro (mantiene valor). De acuerdo con Baur y Lucey (2010), y con Ratner y Chiu (2013) un activo se puede usar para diversificación si mantiene una correlación positiva débil con otro activo en promedio. Recordemos que la volatilidad de estos activos financieros es muy alta y esa es una oportunidad para los especuladores o tomadores de riesgo. Sin embargo, dependiendo del periodo de tiempo, no necesariamente la alta volatilidad es debida a la especulación con éstos activos. Lo anterior está publicado en algunos artículos de la literatura. Falta esperar la evolución de este interesante mercado que combina tecnología, finanzas y características que no han permitido definir qué tipo de objeto es. Una extensión del trabajo es investigar si la volatilidad alta que caracteriza a este tipo de activos es debido realmente a especulación, o bajo qué condiciones es consecuencia de la especulación. Análogamente, si también la existencia de burbujas en este mercado nos indicaría que la naturaleza de estos activos es especulativa, y si eso es lo que mantiene el mercado activo.

Otros estudios han publicado que existe correlación cero de los rendimientos del Bitcoin con otros activos comunes como las acciones, bonos, commodities etc. Esta es otra ventana de oportunidad, puesto que se puede estudiar la correlación de otras criptomonedas con éstos activo más comunes, a partir de una distribución multivariada. 
REMEF (The Mexican Journal of Economics and Finance)

Estimación de la distribución multivariada de los rendimientos de los tipos de cambio

\section{Referencias}

Barndorff, O. (1977). Exponentially decreasing distributions for the logarithm of the particle size. Proceedings of the Royal Society, Series A, Mathematical and Physical Sciences, 353 (1674), 401-419. doi: 10.1098/rspa.1977.0041

Barndorff, O., Mikosch, T. Resnick, S. (2012). Lévy processes: theory and applications, Springer Science Business Media, New York.

Baur, D.G. and Lucey, B.M. (2010). Is gold a hedge or a safe haven? An analysis of stocks, bonds and gold. Financial Review 45, pp 217-229.

Baur, D., Dimpfl, T. and Kuck, K. (2017). Bitcoin, Gold and the US Dollar- A replication and Extension, Finance Researcher Letters, 25, pp.103-110

Baur, D., Hong, K., Lee, A. (2018). Bitcoin: Medium of exchange or speculative assets? Journal of International Financial Markets, Institutions Money,54, pp.177-189

Bouri, E., Gil-Alana, L.A., Gupta, R., and Roubaud, D. (2016). Modelling Long Memory Volatility in the Bitcoin Market: Evidence of Persistence and Structural Breaks. University of Pretoria, working paper, 2016-54.

Breymann, W. Lüthi, D. (2013). ghyp: A package on generalized hyperbolic distributions. Available in: http://cran.r-project.org/web/packages/ghyp/vignettes/Generalized ${ }_{H}$ yperbolic $_{D}$ istribution.pdf

Cermak, Vavrinec. (2017). Can Bitcoin become a viable alternative to fiat currencies? An empirical analysis of Bitcoin's volatility based on a GARCH model. Available online: https://ssrn.com/ abstract $=2961405$.

Cont, R. (2001). Empirical properties of asset returns: stylized facts and statistical issues. Quantitative Finance, 1, 223-236.

Corbet, S., Lucery, B.M., Urquhart, A., Yarovaya, L. (2018). Cryptocurrencies as a financial asset. A systematic analysis, doi:10.2139/ssrn.3143122.

Corsi, F., and Sornette, D. (2014). Follow the money: The monetary roots of bubbles and crashes. International Review of Financial Analysis, 32, 47-59.

Demir, E., Goznor, G., Lau. C.K.M., Vinge, S.A. (2018). Does economic policy uncertainty predict the bitcoin returns? An empirical investigation. Finance Research Letters, doi:10.1016/j.frl.2018.01.005.

Dwyer, G.P. (2015) The economics of Bitcoin and similar private digital currencies. Journal of Financial Stability. 17, 81-91.

Gandal, N., Halaburda, H. (2016). Can we predict the winner in market with network effects? Competition in cryptocurrency market. Games, 7, 16.

Glaser, F., Zimmermann, K., Haferkorn, M. W. M., Siering, M, (2014). Bitcoin-asset or currency? Revealing users hidden intentions. In: 22nd European Conference on Information Systems (ECIS), Tel Aviv, Israel.

GSR, (1998). E-gold; better money; since 1996 URL: https://web.archive.org/web/19981202142942/ http://www.e-gold.com:80/.outline:access

Henriques I., Sadorsky, P., (2018). Can bitcoin replace gold in an investment portfolio? Journal of Risk Financial Management, 11 (48), doi:10.3390/jrfm11030048.

Chan, Stephen., Jeffrey, Chu., Saralees, Nadarajah., and Joerg, Osterrieder. (2017). A statistical analysis of cryptocurrencies. Journal of Risk Financial Management, 10 (12), doi:10.3390/jrfm10020012.

Chaum, D.L. (1983). Blind signatures for untraceable payments. URL: http://ecash.com/.online:access 07-November-2017.

Chu, Jeffrey, Stephen Chan, Saralees Nadarajah, and Joerg Osterrieder. (2017). GARCH Modeling of Cryptocurrencies, Technical Report. School of Mathematics, University of Manchester, Manchester, UK.

Dyhrberg, Anne Haubo. (2016a). Hedging capabilities of Bitcoin. Is it the virtual gold? Finance Research Letters, 16, 139-44.

Dyhrberg, Anne Haubo. (2016b). Bitcoin, gold and the dollar - A GARCH volatility analysis. Finance Research Letters, 16, 85-92.

Garcia, D., Tessone, C., Mavrodiev, P., and Perony, N. (2014). The digital traces of bubbles: Feedback cycles between socio-economic signals in the Bitcoin economy. Journal of the Royal Society Interface, 11 (99), 1-8.

Glaser, F., Haferhorn, M., Weber, M.C., Zimmarmann, K., and Siering, M.b. (2014). Bitcoin -Asset or currency? Revealing users' hidden intentions. ECIS 2014 Tel Aviv.

Hu, W. (2005). Calibration of Multivariate Generalized Hyperbolic Distributions using the EM Algorithm, with Applications in Risk Management, Portfolio Optimization and Portfolio Credit Risk. Available at: http://fsu.digital.flvc.org/islandora/object/fsu \%3A181953

Konlak, V. and Wilcox D. (2014). A Comparison of Generalized Hyperbolic Distribution Models for Equity Returns. Journal of Applied Mathematics Volume 2014, Article ID 263465.

Kristoufek, L. (2013). Bitcoin meets Google Trends and Wikipedia: Quantifying the relationship between phenomena of the Internet era. Scientific Reports, 3, 3415

McAssey, M. (2013). An empirical goodness-of-fit test for multivariate distributions. Journal of Applied Statistics, 40 (5), 1120-1131. 
Paolella, M. (2007). Intermediate Probability. John Wiley Sons, West Sussex, England.

Protassov, R. (2004). EM-based maximum likelihood parameter estimation for multivariate generalized hyperbolic distributions with $\lambda$ fixed. Statistics and Computing, 14 (1), 67-77.

Ratner, M. and Chiu, C. (2013) Hedging Stock Sector Risk with Credit Default Swaps. International Review of Financial Analysis.30, pp 18-25.

Ross, S. (2012). Simulation, Academic Press, USA.

Sahoo P. (2017) Bitcoin as digital money: Its growth and future sustainability. Theoretical and Applied Economics. 4(613), pp. 53-64.

Shuyue (2018). Volatility connecedness in the cryptocurrency market. Is Bitcoin a dominant cryptocurrency? doi:10.1016/J.IRFA.2018.08.013

Tsay, R. (2010). Analysis of financial time series, Wiley, Cambridge, Massachusetts.

Xin, L. Chong A. (2016). The technology and economic determinants of cryptocurrency exchange rates: determinants of cryptocurrency exchange rates: The case of Bitcoin, Decision Support Systems, doi: $10.1016 /$ j.dss.2016.12.001

Yermack, D. (2013). Is Bitcoin a real currency? An economic appraisal. National Bureau of Economic Research, (No. w19747). 